\title{
The Influence of Cardiac Ablation on the Electrophysiological Characterization of Rat Isolated Atrium: Preliminary Analysis
}

\author{
JS Paredes ${ }^{1}$, S Pollnow ${ }^{2}$, O Dössel ${ }^{2}$, J Salinet $^{1}$ \\ ${ }^{1}$ Biomedical Engineering, Engineering, Modelling and Applied Social Sciences Centre of the Federal \\ University of ABC, São Paulo, Brazil \\ ${ }^{2}$ Institute of Biomedical Engineering (IBT), Karlsruhe Institute of Technology (KIT), Karlsruhe, \\ Germany
}

\begin{abstract}
Atrial fibrillation $(A F)$ is the most common cardiac arrhythmia seen in clinical practice and its treatment by antiarrhythmic drugs is still non-effective. Radiofrequency catheter ablation (RFA) has been widely accepted as a strategy to prevent $A F$ by creating myocardial lesions to block the AF electrical wavefront propagation and eliminate arrhythmogenic tissue. In this study, we analyzed the electrophysiological impact of different RFA time duration strategies through a controlled animal protocol. Electrical activity of the isolated right atrium of rats, under different RFA time strategies on the epicardium, was acquired during $4 s$ on the endocardium by electrical Mapping (EM) and simultaneously on the endocardium by Optical Mapping $(O M)$, respectively. Analyses were concentrated on both time and frequency domain, through analysis of signal's morphology, local activation time, conduction velocity, dominant frequency $(D F)$, and organization index (OI). The morphology of the optical and electrical signals was altered as the ablation time increased, making it difficult to identify activation times. Moreover, DF and OI decreased with increasing ablation time implied in fragmented electrograms. Through the characterization of traditional metrics applied to the electrical and optical data, it was possible to identify important changes, in time and frequency, inside the ablated regions.
\end{abstract}

\section{Introduction}

$\mathrm{AF}$ is the most common cardiac arrhythmia, affecting $1-2 \%$ of the worldwide population [1]. It is characterized by the collapse of the synchronized wavefront atria depolarization, experiencing disorganized and self-sustaining electrical activation patterns [1]. During AF, the atria do not empty well between contractions, leading to formation of blood clots and thromboembolic events, increasing the risk of stroke five-fold and doubling mortality [1].
Three different theories were postulated in an attempt to explain the mechanism of AF: multiple and continuous intra-atrial reentries, focal, and rotor [2,3]. Occurrences vary individually between patients, and multiple can coexist simultaneously, or intercalated with each other in the same patient, favoring the complex heart rate [2].

The treatment by antiarrhythmic drugs is still non-effective and RFA has been widely accepted as a strategy to prevent AF [4]. This approach aims to create myocardial lesions to block the AF electrical wavefront propagation and eliminate arrhythmogenic tissue [4]. The primary ablation strategy for $\mathrm{AF}$ involves electrical isolation of the pulmonary veins (PVs), aiming to restore and maintain sinus rhythm [4]. Such a technique electrically disconnects the PVs from the rest of the atria, preventing the spread of self-exciting electrical activity to the left atrium $[1,4]$.

Recurrence of AF after RFA occurs in at least $20-40 \%$ of patients, where conduction in the cauterized tissue is recovered [5]. Durable PV isolation may be difficult to achieve, and electrical isolation is more likely to be permanent after a repeat ablation procedure [5]. Assessment of traditional electrophysiological characteristics of the ablated cells allows for inference on its excitability and conductivity state and helps to further understand the RFA role. This study aims at analyzing the electrophysiological impact of different RFA time duration strategies through a controlled animal protocol, mimicking clinical situations.

\section{Methods}

\subsection{Experimental protocol}

Experiments were carried out in agreement with the European Council guidelines for the care and use of laboratory animals and have been approved by the local Committee for Animal Welfare (35-9185.81/G-61/12). For this study, 2 adult Fisher rats (male, 400-450 g, 4 months old) were used. Firstly, the right atrium is dissected by cut- 
ting along the tricuspid valve to the superior vena cava (10 $\mathrm{x} 10 \mathrm{~mm}$ ) followed by a bath with a Krebs-Henseleit solution under a controlled temperature of $36.7^{\circ} \mathrm{C} \pm 0.5^{\circ} \mathrm{C}$. The sinoatrial node is ablated and the epicardium stimulated at 6.7 Hz. RFA was performed with a tungsten microdissection electrode connected to the electrosurgical unit (MD1, Micromed, Medizintechnik GmbH, Germany) in the center of the atrium epicardium. The RFA strategy is performed as follows: the same area of the tissue is ablated during $0.5 \mathrm{~s}, 1.5 \mathrm{~s}, 2 \mathrm{~s}, 2.5 \mathrm{~s}, 3 \mathrm{~s}$, and $4 \mathrm{~s}$ respectively. Before and after each strategy, epicardium atrium unipolar electrograms (EMGs) and Optical Activation Potentials (OAPs) are recorded simultaneously, waiting for $2 \mathrm{~min}$ intervals between strategies. By the end, the epicardium has an accumulative ablation for $14.5 \mathrm{~s}$ [6].

\subsection{Optical and Electrical Mapping}

The dye Di-4-ANEPPS (Sigma Aldrich, St. Louis, USA) was used to record relative changes of transmembrane voltage. Excitation was performed with two LEDs (525 nm) supplied with $4 \mathrm{~A}$ and connected with two narrow-band filters of $530 \mathrm{~nm}$. After that, the light was directed to a glass diffuser (diameter 2", 600 grit) and then focused by two plane-convex lenses (diameter 2", focal lengths: $150 \mathrm{~mm}$ ), propagating to a Dichroic mirror and a Makro-Planar before reaching the endocardium. After excitation, light is propagated back to the Makro-Planar and Dichroic mirror, followed by a long-pass filter $(610 \mathrm{~nm})$ and the high-speed focal length lens. Fluorescence was then guided to a CCD camera that acquires $82 \times 82$ pixels sampled at $868 \mathrm{~Hz}$ with a binning factor of 2 . The region of the camera is approximately $10.5 \times 10.5 \mathrm{~mm}$ with a spatial resolution of $128 \mu \mathrm{m} \times 128 \mu \mathrm{m}$. For each pixel, the emission spectra shift over time allowing detection of the optical action potentials (OAPs). The matrix is reduced to $64 \times 64$ pixels due to noise on the borders. Simultaneously to optical mapping, epicardium atrium electrograms (EGMs) were recorded with a circular multielectrode array (MEA) containing eight silver/silver chloride electrodes (3.5 mm of diameter) with a sampling frequency of 100 $\mathrm{kHz}$. We assumed that the transmural endo-epi tissue has no influence on the acquired OAPs and EGMs. The assessment of traditional electrophysiological parameters are compared across the epicardium and endocardium.

\subsection{Electrophysiological characterization}

Preprocessing: OAPs were embedded in 3D matrices (64x64 pixels, $4 \mathrm{~s}$ ). For each OAP, the baseline (BL) was estimated with a 1D Gaussian low-pass filter with a cutoff frequency of $2 \mathrm{~Hz}$ and then subtracted from its raw signal [7]. EGMs were downsampled to $5 \mathrm{kHz}$ to save computational processing time and memory. The pacing artifacts are then canceled as follows: atrial activity is removed with a 3rd-order Butterworth high-pass filter ( $\mathrm{fc}=1$ $\mathrm{kHz}$ ) and pacing peak detection on the surrogate signal is performed. The pacing segment is defined as $2 \mathrm{~ms}$ before and after the time instant of the pacing peak. Its removal is done by subtraction segment on the raw electrical signal. After the pacing far-field removal, the EGMs were band-pass filtered with a 3rd-order Butterworth low-pass ( $\mathrm{fc}=743 \mathrm{~Hz})$ and high-pass $(\mathrm{fc}=0.6 \mathrm{~Hz}$ ) filters. A notch filter centered at $50 \pm 1 \mathrm{~Hz}$ was used to remove the power line noise.

Signal Morphology: 10 OAPs were selected (8 underneath the MEA's electrodes, 1 far away from the ablated area, and 1 in the center of the RFA area). The comparison of the morphology between OAPS recorded at baseline and after each RFA time strategy was performed with Pearson Correlation (PC). The analysis was also expanded to the EGMs' morphology.

Time analysis: Local activation time (LAT) of the OAPs was obtained with the maximum positive derivative $(+\mathrm{dI} / \mathrm{dt})$ calculated during $4 \mathrm{~s}$ for the pixels underneath the MEA's electrodes [6] and the differences between maximal and minimal LATs were calculated. The mean dispersion (LAT) was calculated as the mean value of the obtained differences. With the LATs, the isochronous maps are generated and the conduction velocity $(\mathrm{CV})$ calculated through the Euclidean distance between the earliest and most delayed activated areas. For each array after the different RFA times, a mean OAP per pixel is generated with all OAPs detected during 4 seconds. Normalization is performed per protocol prior calculation of action potential duration (APD) obtained between the time shift from the LAT to the time where the amplitude decays 30, 50, and $70 \%$ from highest OAP positive peak [8]. The LATs from EGMs were obtained with the minimum downstroke derivative $(-\mathrm{dV} / \mathrm{dt})$ [6], after that time differences of the LATs are calculated.

Frequency analysis: Fast Fourier Transform (FFT) is applied to estimate the frequency spectrum. A Hamming window and zero-padding factor of 5 was applied when performing the FFT (frequency step of $0.05 \mathrm{~Hz}$ ). Dominant frequncy (DF) is identified, defined as the highest frequency peak power within the $4-12 \mathrm{~Hz}$ range. The respective organization index (OI) is also calculated.

\section{Results}

\subsection{Morphology:}

Figure 1A shows two consecutive OAPs recorded at the center of the ablated area during baseline (left), $2.5 \mathrm{~s}$ (middle), and $4 \mathrm{~s}$ (right) of ablation, with their respective LAT 
detected. The OAPs do not show a well-defined activation morphology and increased levels of fractionation according to the increased time of RFA, making it difficult for LAT detection. Figure 1B shows only an OAP, acquired underneath electrode 3 of the MEA, highlighting lower fractionation levels. Figure 1C shows the respective EGM for electrode 3, where the most noticeable change was the magnitude of the minimum amplitude, that after $2.5 \mathrm{~s}$ of ablation disappears completely, showing that the tissue underneath the electrode was electrically damaged and the wavefront is not receding from it.

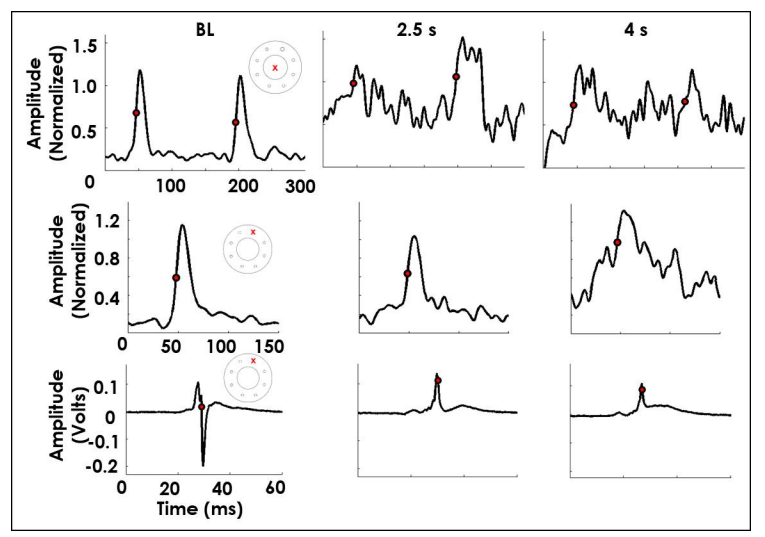

Figure 1. A. OAPs at baseline (left), $2.5 \mathrm{~s}$ (middle) and $4 \mathrm{~s}$ after RFA (right) obtained at the ablated area. B. OAP acquired underneath MEA electrode 3. C. Respective EGM for electrode 3 .

Table 1 shows correlation values for the 8 OAPs underneath the MEA's electrodes, one OAP at the center, and far away from the ablated area, across the different time RFA strategies. It's possible to see values of high correlation up to $2.5 \mathrm{~s}$ for experiment $\mathrm{A}$ and up to $4 \mathrm{~s}$ for experiment $\mathrm{B}$. The OAPs recorded far away from the ablated area were not affected in terms of morphology. The table also shows the outcomes for the EGMs. Overall, their morphology is highly affected. A high correlation is seen only in case A for a RFA of $0.5 \mathrm{~s}$.

Table 1. OAPs and EGMs's Pearson Correlation.

\begin{tabular}{lllllll}
\hline \multicolumn{3}{c}{ Exp A } & \multicolumn{5}{c}{ Exp B } \\
\hline time & mean \pm SD & in & out & mean \pm SD & in & out \\
\hline \multicolumn{7}{c}{ OAP } \\
\hline $0.5 \mathrm{~s}$ & $0.94 \pm 0.02$ & 0.93 & 0.94 & $0.88 \pm 0.04$ & 0.87 & 0.95 \\
$2.5 \mathrm{~s}$ & $0.88 \pm 0.09$ & 0.74 & 0.95 & $0.86 \pm 0.05$ & 0.65 & 0.94 \\
$4 \mathrm{~s}$ & $0.01 \pm 0.02$ & 0.00 & -0.02 & $0.72 \pm 0.16$ & 0.45 & 0.95 \\
\hline \multicolumn{7}{c}{ EGM } \\
\hline $0.5 \mathrm{~s}$ & $0.95 \pm 0.04$ & & & $0.41 \pm 0.32$ & & \\
$2.5 \mathrm{~s}$ & $0.46 \pm 0.34$ & & $0.16 \pm 0.32$ & & \\
$4 \mathrm{~s}$ & $0.05 \pm 0.03$ & & & $0.19 \pm 0.29$ & & \\
\hline
\end{tabular}

\subsection{Time Analysis}

The $\triangle$ LAT time across the OAPs recorded underneath the MEAs electrodes increased (in $\mathrm{ms}$ ) from $4.35 \pm 0.06$ (baseline) to $9.44 \pm 2.56$ (2.5 s) and $64.8 \pm 81.63$ (4 s). The EGMs presented a similar trend, increasing from 6.0 \pm 1.2 to $11.7 \pm 2.5$ and $12.6 \pm 2.7$, respectively.

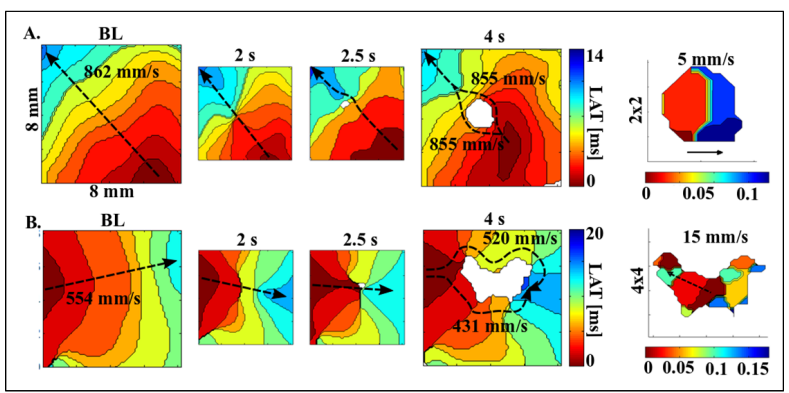

Figure 2. A. LAT map generated at baseline and after $2 \mathrm{~s}$, $2.5 \mathrm{~s}$ and $4 \mathrm{~s}$ of RFA. B. Similar analysis for experiment B.

Figure 2A shows the isochronous maps generated in baseline and after 2, 2.5 and $4 \mathrm{~s}$ (case A). In baseline, the earliest activation starts at the bottom right and the latest activation at the upper left. A similar behavior is seen up to $2 \mathrm{~s}$ of RFA, however after a RFA of 2.5 , s the tissue shows a great reduction of wavefront propagation around the ablated area. The CV kept around $860 \mathrm{~mm} / \mathrm{s}$ at areas away from the ablated regions (Fig. 2A). After $4 \mathrm{~s}$ of RFA the ablated area shows a CV of $4.92 \mathrm{~mm} / \mathrm{s}$ (Fig. 2A right). We can see that the ablated area started to act as a functional block contributing to formation of entries. Figure $2 \mathrm{~B}$ shows the similar analysis for experiment $\mathrm{B}$. The earliest activation starts at the left side of the figure and the latest activation is at the right side. The $\mathrm{CV}$ during baseline was $554.05 \mathrm{~mm} / \mathrm{s}$. The $\mathrm{CV}$ within the ablated area reduced to $14.75 \mathrm{~mm} / \mathrm{s}$ and outside 520.96 and $431.35 \mathrm{~mm} / \mathrm{s}$ at the RFA of 4 s. Similarly, there was a dramatic CV reduction in experiment B starting after RFA of $2.5 \mathrm{~s}$, indicating loss of electrical conduction from the related cells.

Figure 3 shows the APD changes after different RFA time strategies. It can be seen that APD values up to $2.5 \mathrm{~s}$ increased and then decreased below baseline levels.

\subsection{Frequency Analysis}

Figure 4 shows DF and OI results for both EGMs and OAPs. In the top row of Figure 4A, we can see that DF maps up to RFA of $2.5 \mathrm{~s}$ have large areas of $6.7 \mathrm{~Hz}$. After RFA of $4 \mathrm{~s}$, it decreased to $4.7 \mathrm{~Hz}$, followed by small areas at $9.4 \mathrm{~Hz}$ (yellow), and $5.4 \mathrm{~Hz}$ (green) in the ablated area. On the other hand, EGMs'DF is $4.7 \mathrm{~Hz}$. The respective OI maps are displayed below the DF maps. We can see that the overall OI values for the OAPs close to the ablated area 


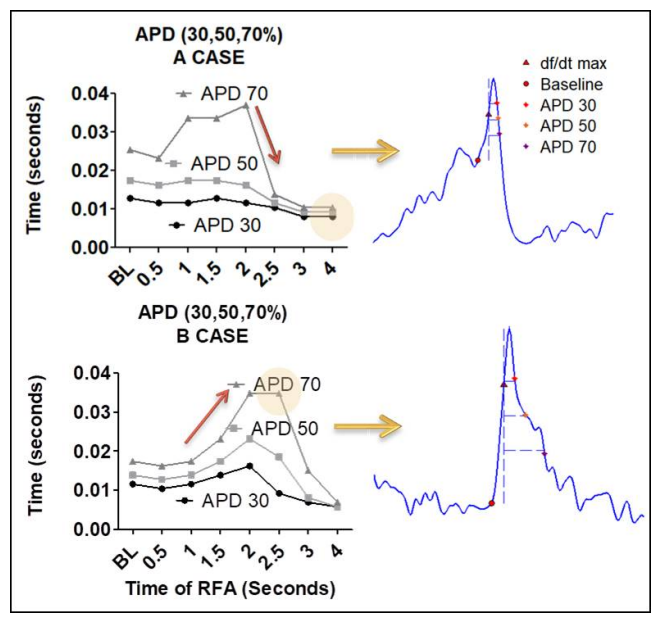

Figure 3. A. APD30,50,70 evolution for each RFA strategy and the respective values for the OAP recorded underneath electrode 5 after RFA of $4 \mathrm{~s}$. B. Similar analysis now for experiment B.

is reduced, in contrast to the EGMs. Figure 4B summarizes the analysis for experiment B. DFs at RFA of $4 \mathrm{~s}$ is around $6.7 \mathrm{~Hz}$ out the ablated area and reduced to $5.7 \mathrm{~Hz}$ at the ablated area. The OI maps have similar patterns.

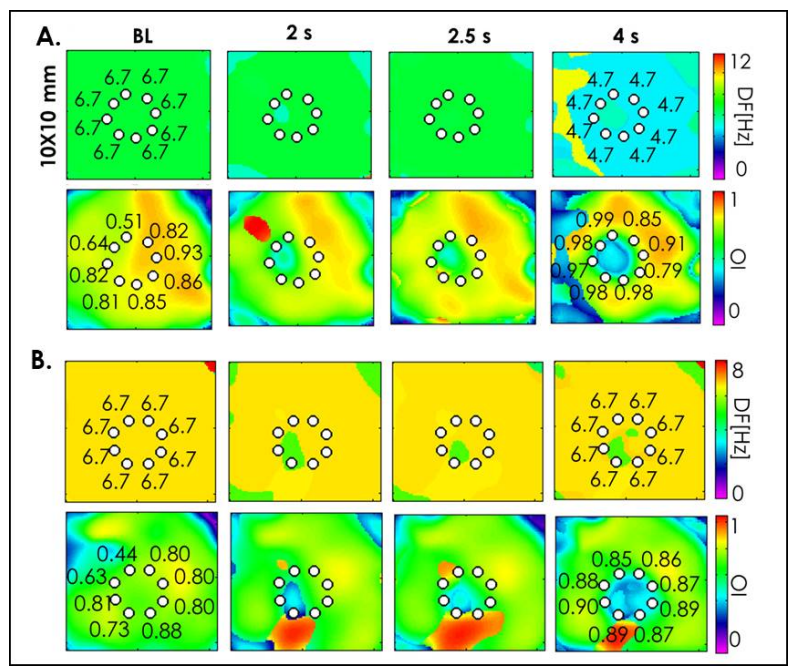

Figure 4. A. At the upper side, the OAP DF maps from baseline to RFA of $4 \mathrm{~s}$ with the respectives DF values from the EGMs. Below, the related OI maps with the OI values for the EGMs. B. Analyses for experiment B.

\section{Discussion}

EGMs and OAPs suffered morphology and amplitude changes, with increased levels of fractionation due to the RFA. It avoids the reliability of activation detection. The lower peak of the EGMs disappears after $2 \mathrm{~s}$ of ablation, which is probably due to the fact that the cells are not being activated, and the wavefront dramatic attenuated. The ablated areas present electrophysiological differences on both time and frequency analysis when compared with areas underneath the contact electrodes area. Changes in the morphology, time and frequency are detected after $2 / 2.5 \mathrm{~s}$ of RFA.

\section{Acknowledgments}

Program of Alliances for Education and Training (Scholarship Brazil - PAEC OEA-GCUB-2017).

\section{References}

[1] January C, Wann L, Calkins H. Guideline for the management of patients with atrial fibrillation: A report of the american college of cardiology. Journal of the American College of Cardiology 2019;74(1):104-32.

[2] Konings K, Kirchhof C, Smeets J, Wellens H, Penn O, Allessie M. High-density mapping of electrically induced atrial fibrillation in humans, volume 89(4). Philadelphia: Circulation, 1994.

[3] Zipes D, Jalife J. Cardiac Electrophysiology From Cell To Bedside. Philadelphia: SAUNDERS, 2004.

[4] Kirchhf P, Eckardt L. Ablation of atrial fibrillation: for whom and how? Heart 2013;96:1325-1330.

[5] Darby A. Recurrent atrial fibrillation after catheter ablation: Considerations for repeat ablation and strategies to optimize success. J Atr Fibrillation 2016;9(1)-1427.

[6] Pollnow S. Characterizing Cardiac Electrophysiology during Radiofrequency Ablation, volume 24. Karlsruhe, Germany: Karlshure Transactions on Biomedical Engineering, 2018.

[7] Pollnow S, Pilia N, Schwaderlapp G. An adaptive spatiotemporal gaussian filter for processing cardiac optical mapping data. Computers in Biology and Medicine 2018; 102:267-277.

[8] Ting YY. An automated system using spatial oversampling for optical mappingin murine atria. development and validation with monophasic andtransmembrane action potentials. Progress in Biophysics and Molecular Biology 2014;1-9.

Address for correspondence:

Jimena Gabriela Siles Paredes

Biomedical Engineering - CECS- UFABC Street: Av.Anchieta, Sao Bernardo do Campo - SP, Brazil

gabriela.siles.paredes@gmail.com 